\title{
Estimación del cardinal del espectro maximal de un producto de cuerpos
}

\section{Estimation of the cardinality of the maximal spectrum of a product of fields}

\author{
C. Granados Pinzón a* \\ W. Olaya León a \\ S. Pinzón Durán a
}

Fecha de Recepción: 27 - mar. - 2017.

Fecha de Aceptación: 18 - abr. - 2018.

\section{Resumen}

En este artículo presentamos propiedades generales de un producto de anillos conmutativos con unidad. Caracterizamos el espectro primo y maximal de una suma de anillos y probamos que el espectro de un producto de cuerpos es $\mathrm{T}_{1}$, o equivalentemente, que es Hausdorff. Por último, estimamos el cardinal del espectro maximal de un producto de cuerpos.

Palabras clave: localización, producto directo de anillos, espectro primo y maximal.

\begin{abstract}
In this paper we show general properties of a product of commutative rings with unity. We obtain a characterization of the prime spectrum of a sum of rings and if we consider a product of fields them its spectrum is $\mathrm{T}_{1}$, or equivalently, it is Hausdorff. Finally we estimate the cardinality of the maximal spectrum of a product of fields.
\end{abstract}

Key words: localization, direct product of rings, prime and maximal spectrum.

a Escuela de Matemáticas, Universidad Industrial de Santander, Bucaramanga, Colombia.

* Correo electrónico: cigranad@uis.edu.co 


\section{Granados Pinzón et. al.}

\section{INTRODUCCIÓN}

Sean $R_{1}$ y $R_{2}$ anillos conmutativos con unidades $\mathrm{e}_{1} \mathrm{y} \mathrm{e}_{2}$ respectivamente. El producto cartesiano $R_{1} \times R_{2}$ tiene estructura de anillo con las operaciones suma y producto componente a componente:

$$
\begin{gathered}
\left(f_{1}, f_{2}\right)+\left(g_{1}, g_{2}\right):=\left(f_{1}+g_{1}, f_{2}+g_{2}\right), \\
\left(f_{1}, f_{2}\right) \cdot\left(g_{1}, g_{2}\right):=\left(f_{1} g_{1}, f_{2} g_{2}\right) .
\end{gathered}
$$

Además, $\mathrm{R}_{1} \times \mathrm{R}_{2}$ es un anillo conmutativo con unidad $\mathbf{e}=\left(\mathrm{e}_{1}, \mathrm{e}_{2}\right)$. Todo producto cartesiano, $\mathrm{R}_{1}$ $\times \mathrm{R}_{2}$ tiene asociado sus proyecciones

$$
\begin{aligned}
\pi_{i}: \quad R_{1} \times R_{2} & \rightarrow R_{i} \\
\left(f_{1}, f_{2}\right) & \mapsto f_{i}
\end{aligned}
$$

con $i=1,2$.

Por otra parte, el producto cartesiano $\mathrm{X}_{1} \times \mathrm{X}_{2}$ de los conjuntos $X_{1}$ y $X_{2}$ satisface la propiedad universal: para todo conjunto $\mathrm{Z}$ y para todas las aplicaciones $\varphi_{1}: Z \rightarrow X_{1}$ y $\varphi_{2}: Z \rightarrow X_{2}$, existe una única aplicación $\varphi: Z \rightarrow X_{1} \times X_{2}$ tal que $\varphi_{1}=\pi_{1} \circ \varphi, \varphi_{2}=\pi_{2} \circ \varphi$. El producto cartesiano $R_{1} \times R_{2}$ de los anillos $R_{1}$ y $R_{2}$ satisface la propiedad universal análoga en la categoría de anillos conmutativos. Por tanto, el producto cartesiano de anillos conmutativos es también producto en el sentido de categorías (véase [6]).

Al igual que en conjuntos, podemos considerar el producto cartesiano de una familia de anillos: sea $I$ un conjunto arbitrario y $\left\{R_{i}\right\}_{i \in I}$ una familia de anillos conmutativos con unidad, su producto cartesiano $\prod_{i \in I} R_{i}$ es un anillo conmutativo con unidad con las operaciones suma y producto componente a componente. El producto cartesiano $\prod_{i \in I} R_{i}$ satisface la propiedad universal del producto.

En la categoría de anillos conmutativos se tiene también un producto directo y una suma directa de anillos. Es decir, dada la familia de anillos $\left\{R_{i}\right\}_{i \in I}$, existe un anillo $R=\prod_{i \in I} R_{i}$ junto con los homomorfismos de anillos $\boldsymbol{i}_{i}: R_{i} \rightarrow R, i \in I$, tal que para todo anillo $S$ y para todos los homomorfismos $\varphi_{i}: R_{i} \rightarrow S$, existe un único homomorfismo $\varphi: R \rightarrow S$ con $\varphi_{i}=\varphi \circ \boldsymbol{i}_{i}$ (véase $[1,2,6]$ ).

La suma directa de la familia anterior es el conjunto

$\bigoplus_{i \in I} R_{i}:=\left\{\left(f_{i}\right) \in \prod_{i \in I} R_{i}:\right.$ casi todos los $\left.f_{i}=0\right\}$

donde por "casi todos" queremos decir "todos, excepto un número finito". La suma directa así definida no posee unidad, de tal forma que esta suma no pertenece a la categoría de anillos conmutativos con unidad. En cambio, si se considera el producto tensorial de anillos (vistos como álgebras sobre el anillo de los enteros), entonces este producto tensorial si es el coproducto (también llamado suma directa) en el sentido categórico además las inyecciones canónicas son homomorfismos en la categoría de anillos conmutativos con unidad.

Observe que si el conjunto de índices $I$ es finito, entonces

$$
\bigoplus_{i \in I} R_{i}=\prod_{i \in I} R_{i}
$$

El producto de anillos es un tema de investigación en la actualidad en el área de álgebra conmutativa (véase $[9,10,15,19]$ ). Nuestro interés en este tema se basa en un problema abierto de geometría proyectiva, el cual consiste en caracterizar la recta proyectiva sobre anillos, en particular sobre anillos totales de fracciones. El producto de anillos conmutativos es un anillo total de fracciones (véase [10]). Para alcanzar este fin, en [11] hemos estudiado las $K$-álgebras finitas conmutativas con unidad, pues ellas también son anillos totales de fracciones.

Las rectas proyectivas sobre las $\mathrm{R}$-álgebras bidimensionales $\frac{\mathbb{R}[x]}{\left(x^{2}+1\right)}, \frac{\mathbb{R}[x]}{\left(x^{2}-1\right)}$ y $\frac{\mathbb{R}[x]}{\left(x^{2}\right)}$ generan las tres geometrías clásicas del plano, Moebius, Laguerre y Minkowski (véase [12]). Existen trabajos recientes sobre las rectas proyectivas sobre anillos, pero en general es una teoría muy incompleta. [13] es un trabajo sobre la geometría correspondiente a la $\mathrm{R}$-álgebra tridimensional $\frac{\mathbb{R}[x]}{\left(x^{3}\right)}$ y [10] es un estudio inicial de las rectas proyectivas sobre anillos totales de fracciones, 
sin embargo estudiar la geometría de la recta proyectiva sobre anillos es un problema abierto.

Dado un anillo conmutativo con unidad $R$, podemos asociar a $R$ un espacio topológico

$$
\operatorname{Spec}(R)=\{\mathfrak{p}: \mathfrak{p} \text { es ideal primo de } R\}
$$

llamado el espectro primo de $R$. El espectro primo de un anillo conmutativo relaciona dos áreas de la matemática, el álgebra conmutativa y la topología.

En la sección dos se describe el conjunto de ideales primos y maximales de un producto de anillos, en particular, se muestra que en el caso de productos finitos el espectro de un producto es el producto de los espectros en el sentido topológico. En la tercera sección se estudia el caso particular del producto directo de cuerpos donde usamos el anillo de fracciones por un ideal maximal m, $R_{\mathrm{m}}$. Mostramos que si $R=\prod_{i \in I} R_{i}$ donde $R_{i}$ es cuerpo, entonces los cuerpos $R / \mathfrak{m}$ y $R_{\mathfrak{m}}$, $\mathfrak{m} \in \operatorname{Max}(R)$, son isomorfos. Además, si $R=K^{I}$ con $K$ cuerpo e $I$ finito, entonces los cuerpos $R / \mathfrak{m}$ y $R_{\mathfrak{m}}, \mathfrak{m} \in \operatorname{Max}(R)$, son isomorfos a $K$. De igual forma se tiene este isomorfismo si $R=K^{I}$ con $K$ un cuerpo finito e $I$ un conjunto arbitrario. En la sección cuatro se alcanza el objetivo principal del artículo, el cual consiste en hacer una estimación del cardinal del espectro maximal de un producto infinito de cuerpos para esto se estudian los filtros y ultrafiltros asociados al espectro.

\section{PRELIMINARES}

Sean $I$ un conjunto arbitrario y $\left\{R_{i}\right\}_{i \in I}$ una familia de anillos conmutativos con unidad. Consideremos el anillo producto $R=\prod_{i \in I} R_{i}$ con las operaciones suma y producto componente a componente. Decimos que $f \in R$ es idempotente si $f^{2}=f$. Sea $\pi_{i}: R \rightarrow R_{i}$ la proyección $i$-ésima, es decir para $\boldsymbol{f}=(f(i))_{i \in I} \in R$, $\pi_{i}(\boldsymbol{f})=f(i)$.

Proposición 2.1 Sean $R=\prod_{i \in I} R_{i}$ y $\boldsymbol{f} \in R$. Entonces

(1) $\boldsymbol{f}$ es inversible si y solo si, para todo $i \in I, \pi_{i}(\boldsymbol{f})=f(i)$ es inversible.
(2) $f$ es divisor de cero si y solo si existe $i \in I$ tal que $\pi_{i}(\boldsymbol{f})=f(i)$ es divisor de cero.

(3) $f$ es idempotente si y solo si, para todo $i \in I, \pi_{i}(\boldsymbol{f})=f(i)$ es idempotente.

Demostración. Se sigue de las definiciones.

Corolario 2.2 Sean $R=\prod_{i \in I} R_{i}$ y $\boldsymbol{f} \in R$. Si para todo $i \in I, R_{i}$ es un cuerpo, entonces

(1) $\boldsymbol{f}$ es inversible si y solo si $f(i) \neq 0$ para todo $i \in I$.

(2) $\boldsymbol{f}$ es divisor de cero si y solo si existe $i \in I$ tal que $f(i)=0$.

(3) $\boldsymbol{f}$ es idempotente si y solo si $f(i)$ es cero o uno para todo $i \in I$.

Demostración. Consecuencia de la Proposición 2.1 ya que $R_{i}$ es cuerpo para todo $i \in I$. como

Para cada $j \in I$ definimos $\boldsymbol{e}_{j} \in R=\prod_{i \in I} R_{i}$

$$
\pi_{i}\left(\boldsymbol{e}_{j}\right)=\delta_{i j} 1_{R_{i}}=\left\{\begin{array}{cc}
1_{R_{i}} & \text { si } j=i \\
0 & \text { si } j \neq i
\end{array}\right.
$$

donde $1_{R_{i}}$ es la unidad en $R_{i}$ y $\delta_{i j}$ es la función delta de Kronecker. En la proposición siguiente mostramos las propiedades elementales.

Proposición 2.3 Sean $R=\prod_{i \in I} R_{i}$ y $\boldsymbol{e}_{i} \in R$ con $i \in I$. Entonces

(1) $e_{i}^{2}=e_{i}$.

(2) $\boldsymbol{e}_{i} \cdot \boldsymbol{e}_{j}=\mathbf{0}$ para todo $j \neq i$.

(3) $\boldsymbol{f} \cdot \boldsymbol{e}_{i}=f(i) \boldsymbol{e}_{i}$ para todo $\boldsymbol{f} \in R$.

(4) $\left(\boldsymbol{f}-f(i) \boldsymbol{e}_{i}\right) \cdot \boldsymbol{e}_{i}=\mathbf{0}$ para todo $\boldsymbol{f} \in R$.

Demostración. Son consecuencia directa de la definición de $\boldsymbol{e}_{i}$.

El lema siguiente muestra una equivalencia para un producto finito de anillos. En ([2], pág. 98), podrá encontrar una versión del Lema 2.4 $\mathrm{y}$ otras equivalencias que no trataremos en este trabajo. 
Lema 2.4 Sea $R$ un anillo con unidad $\mathbf{1}_{R} \cdot R$ es producto de una familia finita de anillos si y solo si existen $\boldsymbol{u}_{1}, \ldots, \boldsymbol{u}_{n} \in R$ idempotentes tales que

(1) $\boldsymbol{u}_{i} \cdot \boldsymbol{u}_{j}=\delta_{i j} \boldsymbol{u}_{i}$

(2) $\sum_{i=1}^{n} \boldsymbol{u}_{i}=\mathbf{1}_{R}$.

El conjunto de idempotentes del lema anterior es llamado conjunto ortogonal de idempotentes (véase [2]). Para todos $f, g \in R$ idempotentes se tiene que $f \cdot g$ es idempotente y que $1-f$ es idempotente.

\subsection{Espectro primo y maximal}

Exponemos a continuación algunas definiciones y resultados en el lenguaje de espectros que usaremos más adelante.

Un ideal $\mathfrak{p}$ de un anillo $R$ es primo si $\mathfrak{p} \neq(1)$ y si $a b \in \mathfrak{p}$ entonces $a \in \mathfrak{p}$ o $b \in \mathfrak{p}$, y un ideal $\mathfrak{m}$ de $R$ es maximal si $\mathfrak{m} \neq(1)$ y no existe ningún ideal $\mathfrak{a}$ tal que $\mathfrak{m} \subset \mathfrak{a} \subset$ (1). Esto es equivalente a decir:

$\mathfrak{p}$ es primo si y solo si $R / \mathfrak{p}$ es dominio entero, $\mathfrak{m}$ es maximal si y solo si $R / \mathfrak{m}$ es cuerpo.

Por tanto un ideal maximal es primo pero el recíproco no es cierto en general. El ideal cero es primo si y solo si $R$ es un dominio entero. Cada elemento de $R$ que no es unidad está contenido en un ideal maximal (véase $[4,18]$ ).

A cada anillo $R$ se asocia un espacio topológico

$\operatorname{Spec}(R)=\{\mathfrak{p}: \mathfrak{p}$ es ideal primo de $R\}$

dotado de la topología de Zariski con base de abiertos $\{D(f)\}_{f \in R}$, donde

$$
D(f)=\{\mathfrak{p} \in \operatorname{Spec}(R): f \notin \mathfrak{p}\} .
$$

El complemento de $D(f)$ se llama variedad de $f$,

$V(f)=\operatorname{Spec}(R) \backslash D(f)=\{\mathfrak{p} \in \operatorname{Spec}(R): f \in \mathfrak{p}\}$.

Si $\mathfrak{a}$ es un ideal de $A$, entonces
$V(\mathfrak{a})=\bigcap_{f \in \mathfrak{a}} V(f)=\{\mathfrak{p} \in \operatorname{Spec}(R): \mathfrak{a} \subset \mathfrak{p}\}$.

Se verifican las siguientes propiedades

(1) $V(0)=\operatorname{Spec}(R), V(R)=\emptyset$.

(2) Si $\mathfrak{a} \subset \mathfrak{b}$ son ideales de $R, V(\mathfrak{a}) \supset V(\mathfrak{b})$.

(3) Si $\mathfrak{a}, \mathfrak{b}$ son ideales de $R, V(\mathfrak{a} \cap \mathfrak{b})=V(\mathfrak{a} \mathfrak{b})=$ $V(\mathfrak{a}) \cup V(\mathfrak{b})$.

(4) Si $\{\mathfrak{a}\}_{i \in I}$ es una familia de ideales de $R$, $V\left(\bigcup_{i \in I} \mathfrak{a}_{i}\right)=V\left(\sum_{i \in I} \mathfrak{a}_{i}\right)=\bigcap_{i \in I} V\left(\mathfrak{a}_{i}\right)$.

Note que $D(1)=V(0)=\operatorname{Spec}(R)$ y también que el espacio $\operatorname{Spec}(R)$ es compacto (véase $[4,8,16$, 21]).

En la proposición siguiente vamos a caracterizar el espectro primo y el maximal de una suma directa de anillos conmutativos con unidad.

Proposición 2.5 Sea $R=\prod_{i \in I} R_{i}$ y consideremos la proyección $i$-ésima $\pi_{i}: R \rightarrow R_{i}$ definida por $\pi_{i}(\boldsymbol{f})=f(i)$.

(1) Si para cada $\mathfrak{p} \in \operatorname{Spec}\left(R_{i}\right)$ consideramos $M_{\mathfrak{p}, i}=\pi_{i}^{-1}(\mathfrak{p})$, entonces $M_{\mathfrak{p}, i}$ es un ideal primo de $R$ dado por $M_{\mathfrak{p}, i}=\prod_{j \in I} m_{j}$ con $m_{j}=R_{j}$ para todo $j \neq i$, y $m_{i}=\mathfrak{p}$.

(2) Si para cada $\mathfrak{m} \in \operatorname{Max}\left(R_{i}\right)$ consideramos $M_{\mathfrak{m}, i}=\pi_{i}^{-1}(\mathfrak{m})$, entonces $M_{\mathfrak{m}, i}$ es un ideal $\underset{m a x i m a l}{ }$ de $R$ dado por $M_{\mathfrak{m}, i}=\prod_{j \in I} m_{j}$ con $m_{j}=R_{j}$ para todo $j \neq i, \mathrm{y} m_{i}=\mathfrak{m} .{ }^{j \in I}$

(3) Si $P$ es un ideal primo de $R$ y existe $i \in I$ tal que $\boldsymbol{e}_{i} \notin P$ entonces $\boldsymbol{e}_{j} \in P$ para todo $j \neq i \mathrm{y}$ en consecuencia $i$ es único. Además si $I$ es finito, entonces existe $i \in I$ tal que $\boldsymbol{e}_{i} \notin P$.

(4) Sea $P$ un ideal. Entonces, $\boldsymbol{e}_{i} \in P$ si y solo si $\pi_{i}(P)=R_{i}$.

(5) $\mathrm{Si} I$ es finito, entonces los ideales primos de $R$ son $\operatorname{los} M_{\mathfrak{p}, i} \operatorname{con} \mathfrak{p} \in \operatorname{Spec}\left(R_{i}\right)$.

(6) $\mathrm{Si} I$ es finito, entonces los ideales maximales de $R$ son $\operatorname{los} M_{\mathfrak{m}, i}$ con $\mathfrak{m} \in \operatorname{Max}\left(R_{i}\right)$. 
(7) Si $I$ es finito, entonces

$$
\operatorname{Spec}(R) \simeq \coprod_{i \in I} \operatorname{Spec}\left(R_{i}\right)
$$

(8) $\mathrm{Si} I$ es finito, entonces

$$
\operatorname{Max}(R) \simeq \coprod_{i \in I} \operatorname{Max}\left(R_{i}\right) .
$$

(9) $\mathrm{Si} I$ es infinito, entonces existen ideales maximales y por tanto primos de $R$ que no son de la forma $M_{\mathfrak{m}, i}$.

Demostración. (1) Como $\pi_{i}$ es un homomorfismo de anillos y $\mathfrak{p} \in \operatorname{Spec}\left(R_{i}\right)$, entonces $\pi_{i}^{-1}(\mathfrak{p})=M_{\mathfrak{p}, i}$ es un ideal primo de $R$.

(2) Puesto que $\pi_{i}$ es sobreyectiva y $\mathfrak{m} \in \operatorname{Max}\left(R_{i}\right)$, entonces $\pi_{i}^{-1}(\mathfrak{m})=M_{\mathfrak{m}, i}$ es un ideal maximal de $R$.

(3) Como $\boldsymbol{e}_{i} \cdot \boldsymbol{e}_{j}=\mathbf{0}$ para todo $i \neq j, \boldsymbol{e}_{i} \cdot \boldsymbol{e}_{j} \in P$ y si existe $i \in I$ tal que $\boldsymbol{e}_{i} \notin P$ entonces $\boldsymbol{e}_{j} \in P$ para todo $j \neq i$ luego $\boldsymbol{e}_{i}$ es único. Además, si $I$ es finito y $e_{i} \in P$ para todo $i \in I$ entonces $\sum_{i \in I} \boldsymbol{e}_{i}=\mathbf{1} \in P$ y $P=R$. Por tanto existe $i \in I$ tal que $e_{i} \notin P$.

(4) Sea $P$ un ideal. Si $\boldsymbol{e}_{i} \in P$, entonces $1=\pi_{i}\left(\boldsymbol{e}_{i}\right) \in \pi_{i}(P)$ y como $\pi_{i}$ es sobreyectiva, $\pi_{i}(P)$ es un ideal, luego $\pi_{i}(P)=R_{i}$. Recíprocamente, si $\pi_{i}(P)=R_{i}$, entonces existe $\boldsymbol{a} \in P$ tal que $\pi_{i}(\boldsymbol{a})=1$ luego $\pi_{i}\left(\boldsymbol{e}_{i} \cdot \boldsymbol{a}\right)=1$ y $\pi_{j}\left(\boldsymbol{e}_{i} \cdot \boldsymbol{a}\right)=0$ para todo $j \neq i$ por tanto $\boldsymbol{e}_{i} \cdot \boldsymbol{a}=\boldsymbol{e}_{i}$. Como $\boldsymbol{a} \in P$ se tiene que $\boldsymbol{e}_{i} \in P$.

(5) Si $P$ es un ideal primo de $R$ se tienen dos casos:

(a) existe $i \in I$ tal que $\pi_{i}(P) \neq R_{i}$.

(b) $\pi_{i}(P)=R_{i}$, para todo $i \in I$.

Si se verifica (a), existe $i \in I$ tal que $\pi_{i}(P) \neq R_{i}$, entonces $P=\pi_{i}^{-1}\left(\pi_{i}(P)\right)$.

En efecto, $P \subset \pi_{i}^{-1}\left(\pi_{i}(P)\right)$ y si $\boldsymbol{a} \in \pi_{i}^{-1}\left(\pi_{i}(P)\right)$ entonces $\pi_{i}(\boldsymbol{a}) \in \pi_{i}(P)$ luego existe $\boldsymbol{b} \in P$ tal que $\pi_{i}(\boldsymbol{a})=\pi_{i}(\boldsymbol{b})$ por tanto $(\boldsymbol{a}-\boldsymbol{b}) \cdot \boldsymbol{e}_{i}=\mathbf{0} \in P$.
Como $\boldsymbol{e}_{i} \notin P, \boldsymbol{a}-\boldsymbol{b} \in P$ y en consecuencia $\boldsymbol{a} \in P$ ya que $\boldsymbol{b} \in P$.

Por el ítem (3), el caso (b) no puede darse si $I$ es finito.

(6) Si Mesun ideal maximal de $R$ entonces $M$ es primo y por el ítem (5), $M=M_{\mathfrak{p}, i} \operatorname{con} \mathfrak{p} \in \operatorname{Spec}\left(R_{i}\right)$.

Si $\mathfrak{p}$ no es maximal entonces existe $\mathfrak{m} \in \operatorname{Max}\left(R_{i}\right)$ tal que $\mathfrak{p} \subset \mathfrak{m}$ por tanto $M \varsubsetneqq \pi_{i}^{-1}(\mathfrak{m})$ lo cual es absurdo pues $M$ es maximal de $R$. Entonces p es maximal.

(7) Si se considera en $R=\prod_{i=1}^{n} R_{i}$ los ideales primos, $M_{\mathfrak{p}, i}$. Por el ítem (2),

$$
\operatorname{Spec}(R)=\coprod_{i=1}^{n}\left\{M_{\mathfrak{p}, i}: \mathfrak{p} \in \operatorname{Spec}\left(R_{i}\right)\right\}
$$

Para todo $i$ se define $X_{i}=\left\{M_{\mathfrak{p}, i}: \mathfrak{p} \in \operatorname{Spec}\left(R_{i}\right)\right\}$ se considera la aplicación

$$
\phi_{i}: X_{i} \rightarrow \operatorname{Spec}\left(R_{i}\right)
$$

definida por $\phi_{i}\left(M_{\mathfrak{p}, i}\right)=\pi_{i}\left(M_{\mathfrak{p}, i}\right)=\mathfrak{p}$. Así, $\phi_{i}$ es inyectiva pues si $\mathfrak{p}=\mathfrak{q}$ entonces $M_{\mathfrak{p}, i}=M_{\mathfrak{q}, i}$ y $\phi_{i}$ es sobreyectiva pues $\pi_{i}$ es sobreyectiva, y $\phi_{i}$ es continua ya que $\phi_{i}^{-1}(V(\mathfrak{p}))=\left\{M_{\mathfrak{q}, i} \in \operatorname{Spec}(R)\right.$ : $\mathfrak{p} \subset \mathfrak{q}\}$ y $\left\{M_{\mathfrak{q}, i} \in \operatorname{Spec}(R): \mathfrak{p} \subset \mathfrak{q}\right\}=V\left(M_{\mathfrak{p}, i}\right)$, donde $V(\mathfrak{p})$ denota el cerrado de Zariski del ideal $\mathfrak{p}$. De igual forma, $\phi_{i}^{-1}$ es continua y por tanto $X_{i}$ es homeomorfo a $\operatorname{Spec}\left(R_{i}\right)$. En consecuencia,

$$
\operatorname{Spec}\left(\prod_{i=1}^{n} R_{i}\right) \simeq \coprod_{i=1}^{n} \operatorname{Spec}\left(R_{i}\right) .
$$

(8) La demostración es similar a la del ítem (7), solo lo cambiamos $\mathfrak{p} \in \operatorname{Spec}\left(R_{i}\right)$ por $\mathfrak{m} \in \operatorname{Max}\left(R_{i}\right)$ y $\operatorname{Spec}(R)$ por $\operatorname{Max}(R)$.

(9) Sea $I$ infinito y consideremos

$Q=\left\{\boldsymbol{a}: \exists J \subset I, J\right.$ finito $\left.\mathrm{y} \pi_{i}(\boldsymbol{a})=0 \forall i \notin J\right\}$

es decir, $Q=\bigoplus_{i \in I} R_{i}$. Entonces $Q$ es un ideal de $R$. En efecto, sean $\boldsymbol{a}, \boldsymbol{b} \in Q$ entonces existen $J_{1}, J_{2} \subset I$ finitos tales que $\pi_{i}(\boldsymbol{a})=0$ y $\pi_{j}(\boldsymbol{b})=0$ para todos $i \notin J_{1}$ y $j \notin J_{2}$ respectivamente. Luego para todo $i \notin J_{1} \cup J_{2}, \pi_{i}(\boldsymbol{a}+\boldsymbol{b})=0$ y $J_{1} \cup J_{2}$ es 
finito. Así, $\boldsymbol{a}+\boldsymbol{b} \in Q$. Además, si $\boldsymbol{\lambda} \in R$, entonces $\pi_{i}(\boldsymbol{\lambda} \cdot \boldsymbol{a})=0$ para todo $i \notin J_{1} \mathrm{y}$ como $J_{1}$ es finito, $\boldsymbol{\lambda} \cdot \boldsymbol{a} \in Q$. Por otra parte, como todo ideal está contenido en un ideal maximal entonces existe un ideal maximal $M$ y por tanto un ideal primo de $R$ que contiene a $Q$ y que cumple que $\pi_{i}(M)=\pi_{i}(Q)=R_{i}$ para todo $i \in I$. Lo que contradice que $M$ tiene la forma del ítem (6).

En la Proposición 2.5(9) $Q$ no es primo, por ejemplo, sea $R=A^{\mathbb{N}}$ con $A$ anillo. Sean $a$ y $b$ divisores de cero de $A$ tales que $a b=0$. Si definimos $\pi_{i}(\boldsymbol{a})=a \mathrm{y} \pi_{i}(\boldsymbol{b})=b$ para todo $i=1, \ldots, n$ entonces $\boldsymbol{a} \cdot \boldsymbol{b}=\mathbf{0} \in Q$ pero a $\boldsymbol{a} \notin Q$ y $\boldsymbol{b} \notin Q$.

Proposición 2.6 Si I es finito, entonces los ideales primos minimales de $R$ son los $M_{\mathfrak{p}, i}=\prod_{j \in I} m_{j}$ con $m_{j}=R_{j}$ para todo $j \neq i$, y $m_{i}=\mathfrak{p}$ donde $\mathfrak{p}$ ideal primo minimal de $R_{i}$.

Demostración. Se tiene por la Proposición 2.5(5).

\section{PRODUCTO DE CUERPOS}

Sean $I$ un conjunto arbitrario y $\left\{R_{i}\right\}_{i \in I}$ una familia de cuerpos. Consideremos el anillo producto de cuerpos $R=\prod_{i \in I} R_{i}$.

La proposición siguiente muestra que todo elemento de un producto de cuerpos es producto de un elemento idempotente y un elemento inversible. Esta propiedad también se puede probar definiendo los anillos regulares (o anillos regulares de von Neumann) ya que un cuerpo es un anillo regular y un producto de anillos regulares es anillo regular (véase [20]).

Proposición 3.1 Para todo $f \in R$, existen $\boldsymbol{u}_{f}$ inversible y $\boldsymbol{\alpha}_{f}$ idempotente tal que $\boldsymbol{f}=\boldsymbol{\alpha}_{f} \cdot \boldsymbol{u}_{f}$. Además, $\boldsymbol{\alpha}_{f}$ es único y, $\boldsymbol{\alpha}_{f}=\mathbf{1}$ si y solo si $\boldsymbol{f}$ es inversible.

Demostración. Definimos la aplicación $u_{f}(i)=f(i)$ si $f(i) \neq 0$ y $u_{f}(i)=1$ si $f(i)=0$ y la aplicación $\alpha_{f}(i)=1$ si $f(i) \neq 0$ y $\alpha_{f}(i)=0$ si $f(i)=0$.Así, $\boldsymbol{u}_{f}$ es inversible, $\boldsymbol{\alpha}_{f}$ es idempotente y $\boldsymbol{f}=\boldsymbol{u}_{f} \cdot \boldsymbol{\alpha}_{f}$. Note que $\boldsymbol{u}_{f}$ no es único pues la construcción es válida con $u_{f}(i) \neq 0$ si $f(i)=0$. Además, $\boldsymbol{\alpha}_{f}$ está unívocamente determinado por $\boldsymbol{f}$. En efecto, $f(i)=u_{f}(i) \alpha_{f}(i)$ y $u_{f}(i) \neq 0$ para todo $i$ pues $\boldsymbol{u}_{f}$ es inversible. Luego $f(i)=0$, entonces $\alpha_{f}(i)=0$ y si $f(i) \neq 0$ entonces $\alpha_{f}(i) \neq 0$ y por tanto $\alpha_{f}(i)=1$ ya que $\boldsymbol{\alpha}_{f}$ es idempotente. En particular, $\boldsymbol{\alpha}_{f}=\mathbf{1}$ si y solo si $\boldsymbol{f}$ es inversible.

Para cada ideal $\mathfrak{a}$ de $R$ definimos el conjunto de idempotentes de $\mathfrak{a}$ como

$$
i d(\mathfrak{a})=\{\boldsymbol{f} \in \mathfrak{a}: \boldsymbol{f} \text { es idempotente }\}
$$

En particular $i d(R)=\{\boldsymbol{f} \in R: \boldsymbol{f}$ es idempotente $\}$.

En la Proposición 3.1, como $\boldsymbol{\alpha}_{f} \in i d(R)$ es único, se llama a $\boldsymbol{\alpha}_{f}$ el idempotente asociado a $\boldsymbol{f}$ y es denotado por $i d(\boldsymbol{f})$. Se define así la aplicación

$$
i d: \quad \begin{aligned}
R & \rightarrow R \\
\boldsymbol{f} & \mapsto i d(\boldsymbol{f})
\end{aligned}
$$

y se tienen las propiedades siguientes:

Proposición 3.2 (1) Sean $\boldsymbol{f}, \boldsymbol{g} \in R$ entonces $i d(\boldsymbol{f} \cdot \boldsymbol{g})=i d(\boldsymbol{f}) \cdot i d(\boldsymbol{g})$.

(2) Para todo ideal $\mathfrak{a}$ se tiene que $i d(\mathfrak{a}) \subset \mathfrak{a}$.

(3) Para todo $\boldsymbol{f} \in R, \boldsymbol{f} \in \mathfrak{a}$ si y solo si $i d(\boldsymbol{f}) \in$ $i d(\mathfrak{a})$.

(4) Sean $\mathfrak{a}, \mathfrak{b}$ ideales de $R$. $\mathfrak{a}=\mathfrak{b}$ si y solo si $i d(\mathfrak{a})=i d(\mathfrak{b})$.

Demostración. (1) Por la Proposición 3.1,

$$
\boldsymbol{f} \cdot \boldsymbol{g}=i d(\boldsymbol{f}) \cdot \boldsymbol{u}_{f} \cdot i d(\boldsymbol{g}) \cdot \boldsymbol{u}_{g}
$$

además el producto de idempotentes es idempotente y el de inversibles es inversible luego

$$
\boldsymbol{f} \cdot \boldsymbol{g}=(i d(\boldsymbol{f}) \cdot i d(\boldsymbol{g})) \cdot\left(\boldsymbol{u}_{f} \cdot \boldsymbol{u}_{g}\right)=
$$
$i d(\boldsymbol{f} \cdot \boldsymbol{g}) \cdot \boldsymbol{u}_{f \cdot g}$

donde $i d(\boldsymbol{f} \cdot \boldsymbol{g})=i d(\boldsymbol{f}) \cdot i d(\boldsymbol{g})$ ya que el idempotente es único.

(2) Para todo $\boldsymbol{f} \in R, \boldsymbol{f}=\boldsymbol{\alpha}_{f} \cdot \boldsymbol{u}_{f}$. Luego, si $\boldsymbol{f} \in \mathfrak{a}$ entonces $\boldsymbol{\alpha}_{f}=\boldsymbol{f} \cdot \boldsymbol{u}_{f}^{-1} \in \mathfrak{a}$.

(3) Inmediato. 
(4) Por el item (3).

Proposición 3.3 Sea $\boldsymbol{f} \in R, \boldsymbol{f}$ no es inversible si y solo si existe $\boldsymbol{g} \neq \mathbf{0}$ tal que $\boldsymbol{f} \cdot \boldsymbol{g}=\mathbf{0}$ y $\boldsymbol{f}+\boldsymbol{g}$ es inversible.

Demostración. Si $f$ no es inversible, por la Proposición 3.1, $\boldsymbol{f}=i d(\boldsymbol{f}) \cdot \boldsymbol{u}_{f}$ y $\mathbf{1}-i d(\boldsymbol{f}) \neq \mathbf{0}$. Entonces se define $\boldsymbol{g}=\boldsymbol{u}_{f} \cdot(\mathbf{1}-i d(\boldsymbol{f}))$ y de esta forma, $\boldsymbol{g} \neq \mathbf{0}$,

$$
\boldsymbol{f} \cdot \boldsymbol{g}=\boldsymbol{u}_{f}^{2} \cdot i d(\boldsymbol{f}) \cdot(\mathbf{1}-i d(\boldsymbol{f}))=\mathbf{0}
$$

y $\boldsymbol{f}+\boldsymbol{g}=\boldsymbol{u}_{f}$ es inversible. Recíprocamente como $\boldsymbol{f} \cdot \boldsymbol{g}=\mathbf{0}$ y $\boldsymbol{g} \neq \mathbf{0}, \boldsymbol{f}$ es no inversible.

Proposición 3.4 Sean $\mathfrak{m} \in \operatorname{Max}(R)$ y $\boldsymbol{f} \in R$. Entonces $\boldsymbol{f} \in \mathfrak{m}$ si y solo si existe $\boldsymbol{h} \notin \mathfrak{m}$ tal que $f \cdot h=0$.

Demostración. Si existe $\boldsymbol{h} \notin \mathfrak{m}$ tal que $\boldsymbol{f} \cdot \boldsymbol{h}=\mathbf{0}$ entonces $\boldsymbol{f} \in \mathfrak{m}$ pues $\mathfrak{m}$ es primo. Recíprocamente, si $f \in \mathfrak{m}, f$ no es inversible y por la Proposición 3.3, existe $\boldsymbol{h} \neq \mathbf{0}$ tal que $\boldsymbol{f} \cdot \boldsymbol{h}=\mathbf{0}$ y $\boldsymbol{f}+\boldsymbol{h}$ es inversible entonces $\boldsymbol{h} \notin \mathfrak{m}$ ya que $\boldsymbol{f} \in \mathfrak{m}$ y $\boldsymbol{f}+\boldsymbol{h} \notin \mathfrak{m}$.

La proposición siguiente muestra un isomorfismo entre el cuerpo $R / \mathfrak{m}, \mathfrak{m} \in \operatorname{Max}(R)$ y el anillo de fracciones o localización $R_{\mathfrak{m}}$.

Proposición 3.5 Para todo $\mathfrak{m} \in \operatorname{Max}(R)$, los cuerpos $R / \mathfrak{m}$ y $R_{\mathfrak{m}}$ son canónicamente isomorfos.

Demostración. Consideremos el homomorfismo canónico

$$
\begin{aligned}
\varphi: R & \rightarrow R_{\mathfrak{m}} \\
f & \mapsto \frac{f}{1}
\end{aligned}
$$

Veamos que para todo $\boldsymbol{f} \in R, \boldsymbol{f} \in \mathfrak{m}$ si y solo si $\frac{f}{1}=\mathbf{0}$. En efecto, si $f \in \mathfrak{m}$ entonces $f$ no es inversible y por la Proposición 3.3, existe $\boldsymbol{g} \neq \mathbf{0}$ tal que $\boldsymbol{f} \cdot \boldsymbol{g}=\mathbf{0}$ y $\boldsymbol{f}+\boldsymbol{g}=\mathbf{1}$. Por tanto $\boldsymbol{g} \notin \mathfrak{m}$ y $\frac{f}{1}=0$. Recíprocamente, si $\frac{f}{1}=\mathbf{0}$, entonces existe $\boldsymbol{g} \notin \mathfrak{m}$ tal que $\boldsymbol{f} \cdot \boldsymbol{g}=\mathbf{0}$. Luego $\boldsymbol{f} \cdot \boldsymbol{g} \in \mathfrak{m}$ y por tanto $f \in \mathfrak{m}$. En consecuencia, $\varphi$ induce un homomorfismo inyectivo

$$
\begin{aligned}
\psi: R / \mathfrak{m} & \rightarrow R_{\mathfrak{m}} \\
\boldsymbol{f}+\mathfrak{m} & \mapsto \frac{f}{1}
\end{aligned}
$$

Veamos ahora que $\psi$ es sobreyectivo. Es decir, si para todo $f \in R$ y para todo $\boldsymbol{g} \notin \mathfrak{m}$ existe $\boldsymbol{h} \in R$ tal que $\frac{f}{g}=\frac{h}{1}$ y esto es equivalente a que existe $\boldsymbol{t} \notin \mathfrak{m}$ tal que $(\boldsymbol{f}-\boldsymbol{g} \cdot \boldsymbol{h}) \cdot \boldsymbol{t}=\mathbf{0}$ pero por la Proposición $3.4, \boldsymbol{f}-\boldsymbol{g} \cdot \boldsymbol{h} \in \mathfrak{m}$. Por tanto, hay que demostrar que para todo $f \in R$ y para todo $\boldsymbol{g} \notin \mathfrak{m}$ existe $\boldsymbol{h} \in R$ tal que $\boldsymbol{f}-\boldsymbol{g} \cdot \boldsymbol{h} \in \mathfrak{m}$. Como $\boldsymbol{g} \notin \mathfrak{m}$, entonces $\boldsymbol{g}+\mathfrak{m} \neq \mathbf{0}$ en el cuerpo $R / \mathfrak{m}$ luego existe $s+\mathfrak{m} \in R / \mathfrak{m}$ tal que $(\boldsymbol{g}+\mathfrak{m})$. $(s+\mathfrak{m})=\mathbf{1}+\mathfrak{m}$ y esto es equivalente a que $\boldsymbol{1}-\boldsymbol{g} \cdot \boldsymbol{s} \in \mathfrak{m}$. Por tanto, $\boldsymbol{f}-\boldsymbol{g} \cdot(\boldsymbol{f} \cdot \boldsymbol{s}) \in \mathfrak{m}$.

Proposición 3.6 Si $\mathfrak{p}$ es un ideal primo de $R$ entonces $\mathfrak{p}$ es maximal.

Demostración. Supongamos que $\mathfrak{p}$ es un ideal primo contenido estrictamente en un ideal maximal $\mathfrak{m}$, entonces existe $\boldsymbol{f} \in \mathfrak{m}$ tal que $\boldsymbol{f} \notin \mathfrak{p}$. Como $f$ no es inversible, por la Proposición 3.3, existe $\boldsymbol{g} \neq \mathbf{0}$ tal que $\boldsymbol{f} \cdot \boldsymbol{g}=\mathbf{0}$ y $\boldsymbol{f}+\boldsymbol{g}$ es inversible. Por tanto, $\boldsymbol{f} \cdot \boldsymbol{g} \in \mathfrak{p}$ y como $\boldsymbol{f} \notin \mathfrak{p}, \boldsymbol{g} \in \mathfrak{p}$. Pero $\mathfrak{p} \subset \mathfrak{m}$ entonces $\boldsymbol{f}+\boldsymbol{g} \in \mathfrak{m}$ y $\boldsymbol{f}+\boldsymbol{g}$ es inversible, entonces $\mathfrak{m}=R$.

En consecuencia, si $R$ es un producto de cuerpos, entonces

$$
\operatorname{Max}(R)=\operatorname{Spec}(R)
$$

Es decir, el espectro de un producto de cuerpos es $T_{1}$, equivalentemente, es Hausdorff. Por otra parte, se puede afirmar que $\mathrm{R}$ tiene dimensión de Krull cero (véase $[2,4,5,7,17]$ ).

La Proposición 3.6 también se puede demostrar utilizando herramientas de anillos con dimensión de Krull cero o anillos 0-dimensionales ya que un cuerpo es 0 -dimensional y el producto de cuerpos es 0 -dimensional (véase [3]). Además el resultado de la Proposición 3.6 no es cierto para el producto de anillos conmutativos, para estudiar este caso se puede ver [9].

Corolario 3.7 Sean $K$ un cuerpo, I un conjunto finito y $R=K^{I}$. Entonces 


\section{Granados Pinzón et. al.}

(1) $\operatorname{Spec}(R)=\operatorname{Max}(R)=\left\{\mathfrak{m}_{i}\right\}_{i \in I}$ donde $\mathfrak{m}_{i}=$ $\{\boldsymbol{f} \in R: f(i)=0\}$,

(2) $R / \mathfrak{m}_{i} \simeq R_{\mathfrak{m}_{i}} \simeq K$ para todo $i$.

Demostración. (1) Es inmediato ya que, por la Proposición 2.5 (6), todos los ideales maximales de $R$ son de la forma $\left\{\mathfrak{m}_{i}\right\}_{i \in I}$ donde $\mathfrak{m}_{i}=$ $\{\boldsymbol{f} \in R: f(i)=0\}$.

(2) Por la Proposición 3.5, basta mostrar que para todo $\mathfrak{m} \in \operatorname{Max}(R), R / \mathfrak{m} \simeq K$. Pero para todo $i \in I$ el homomorfismo $: R \rightarrow K$ definido por $\psi(\boldsymbol{f})=f(i)$ es sobreyectivo y $\operatorname{Ker}(\psi)=\mathfrak{m}_{i}$ entonces

$$
\begin{aligned}
\varphi: \quad R / \mathfrak{m}_{i} & \rightarrow \quad K \\
\boldsymbol{f}+\mathfrak{m}_{i} & \mapsto f(i)
\end{aligned}
$$

es un isomorfismo.

Proposición 3.8 Sean $K$ un cuerpo finito, $I$ un conjunto arbitrario y $R=K^{I}$. Para todo $\mathfrak{m} \in \operatorname{Max}(R)$,

$$
R_{\mathfrak{m}} \simeq R / \mathfrak{m} \simeq K
$$

Demostración. Por la Proposición 3.5, basta demostrar que $R / \mathfrak{m} \simeq K$ para todo $\mathfrak{m} \in \operatorname{Max}(R)$. Sean $K=\left\{\alpha_{0}, \ldots, \alpha_{n}\right\}$ y consideremos la aplicación

$$
\begin{aligned}
\phi: K & \rightarrow R / \mathfrak{m} \\
\alpha & \mapsto \alpha \mathbf{1}+\mathfrak{m}
\end{aligned}
$$

$\phi$ es inyectiva ya que si $\alpha \in K$ y $\alpha \mathbf{1} \in \mathfrak{m}$ entonces $\alpha=0$ pues si $\alpha \neq 0$, por la Proposición 2.1, $\alpha \mathbf{1}$ es inversible en $R$. Veamos que $\phi$ es sobreyectiva. Sea $\boldsymbol{f} \in R$, para todo $i \in I, f(i) \in$ $\left\{\alpha_{0}, \ldots, \alpha_{n}\right\} \mathrm{y}$

$$
\left(\boldsymbol{f}-\alpha_{0} \mathbf{1}\right) \cdots\left(\boldsymbol{f}-\alpha_{n} \mathbf{1}\right)=\mathbf{0}
$$

ya que para todo $i \in I$ existe $j \in\{0,1, \ldots, n\}$ tal que $f(i)=\alpha_{j}$, es $\operatorname{decir}\left(\boldsymbol{f}-\alpha_{j} \mathbf{1}\right)(i)=0$. Entonces

$$
\left(f-\alpha_{0} \mathbf{1}\right) \cdots\left(f-\alpha_{n} \mathbf{1}\right) \in \mathfrak{m}
$$

y por tanto existe $j \in\{1, \ldots, n\}$ tal que $\boldsymbol{f}-\alpha_{j} \mathbf{1} \in \mathfrak{m}$. Así $\phi$ es sobreyectiva.
En la siguiente sección se muestra que el resultado anterior no es cierto si $K$ es un cuerpo infinito, $I$ un conjunto infinito y $R=K^{I}$.

\section{FILTROS Y ULTRAFILTROS DE I}

Hemos visto en la Proposición 2.5(9) que existen ideales maximales de $K^{I}$, con $I$ arbitrario, que no son de la forma $\mathfrak{m}_{i}$. Sobre estos ideales hay mucha literatura (véase $[15,19])$, pero aquí nos limitaremos a estimar el cardinal del conjunto que forman usando filtros y ultrafiltros.

Sean $R=K^{I}, I$ un conjunto arbitrario y $K$ un cuerpo. Para todo $C \subset I$, definimos la aplicación

$$
\begin{aligned}
e_{C}: I & \rightarrow K \\
i & \mapsto e_{C}(i)= \begin{cases}0, & \text { si } i \in C \\
1, & \text { si } i \notin C\end{cases}
\end{aligned}
$$

Proposición 4.1 Sean $B, C \subset I$. Entonces se tiene que:

(1) $e_{I}=0, e_{\emptyset}=1$.

(2) $e_{B}+e_{C}=e_{B \cap C}+e_{B \cup C}$.

(3) $e_{B} \cdot e_{C}=e_{B \cap C}$.

(4) $e_{C}^{2}=e_{C}$.

Demostración. Consecuencia directa de la definición de $e_{C}$.

Veamos que existe una correspondencia biunívoca entre el conjunto de partes de $I, \mathcal{P}(I)$, y el conjunto de los elementos idempotentes de $R, i d(R)$.

Proposición 4.2 Sean $I$ un conjunto arbitrario y $R=K^{I}$. Entonces la aplicación

$$
\begin{aligned}
\gamma: \mathcal{P}(I) & \rightarrow i d(R) \\
C & \mapsto \boldsymbol{e}_{C}
\end{aligned}
$$

es una biyección.

Demostración. $\gamma$ es inyectiva pues si $\mathrm{e}_{B}=\mathrm{e}_{C}$ entonces $e_{B}(i)=e_{C}(i)$, para todo $i \in I$. Luego $e_{B}(j)$ $=0$ con $j \in B$ si y solo si $e_{C}(j)=0$ con $j \in C$. Por tanto $B=C$. Además, $\gamma$ es sobreyectiva pues si $\boldsymbol{f} \in i d(R), f(i)=0$ o $f(i)=1$, para todo 
$i \in I$, entonces $C=\{i \in I: f(i)=0\}$ cumple que $\gamma(C)=\boldsymbol{f}$.

Definición 4.3 Un filtro $\mathfrak{F}$ sobre un conjunto $I$ es una familia no vacía de subconjuntos no vacíos de $I$, que satisfacen:

(1) Si $B, C \in \mathfrak{F}$ entonces $B \cap C \in \mathfrak{F}$,

(2) si $C \in \mathfrak{F}$ y $C \subset D$ entonces $D \in \mathfrak{F}$.

Proposición 4.4 Si a es un ideal propio de $R$, entonces

$$
\begin{gathered}
\mathfrak{F}(\mathfrak{a})=\left\{C \subset I: \boldsymbol{e}_{C} \in \mathfrak{a}\right\}=\gamma^{-1}(i d(\mathfrak{a})) \text { es un } \\
\text { filtro en } I .
\end{gathered}
$$

Demostración. Veamos que $\mathfrak{F}(\mathfrak{a})$ cumple las condiciones de filtro. Puesto que $e_{I}=0 \in \mathfrak{a}$, entonces $I \in \mathfrak{F}(\mathfrak{a})$ y por tanto $\mathfrak{F}(\mathfrak{a})$ es no vacío. Ahora si $B, C \in \mathfrak{F}(\mathfrak{a})$ entonces $e_{B}, e_{C} \in \mathfrak{a}$ y como $\mathfrak{a}$ es ideal, $e_{B}+e_{C}-e_{B} \cdot e_{C}=$ $\boldsymbol{e}_{B \cap C} \in \mathfrak{a}$, por tanto $B \cap C \in \mathfrak{F}(\mathfrak{a})$. Por último, si $C \in \mathfrak{F}(\mathfrak{a})$ y $C \subset D$ entonces $\boldsymbol{e}_{C} \cdot \boldsymbol{e}_{D}=$ $\boldsymbol{e}_{C \cup D}=\boldsymbol{e}_{D} \in \mathfrak{a}$. Luego $D \in \mathfrak{F}(\mathfrak{a})$.

Lema 4.5 Sean $\mathfrak{a}$ y $\mathfrak{b}$ ideales de $R$. Entonces $\mathfrak{a} \subset \mathfrak{b}$ si y sólo si $\mathfrak{F}(\mathfrak{a}) \subset \mathfrak{F}(\mathfrak{b})$.

Demostración. Sea $C \subset I$ tal que $C \in \mathfrak{F}(\mathfrak{a})$ entonces $\boldsymbol{e}_{C} \in \mathfrak{a} \subset \mathfrak{b}$, luego $\boldsymbol{e}_{C} \in \mathfrak{b}$ por tanto $C \in \mathfrak{F}(\mathfrak{b})$. Recíprocamente, si $\boldsymbol{e}_{C} \in \mathfrak{a}$ entonces $C \in \mathfrak{F}(\mathfrak{a}) \subset \mathfrak{F}(\mathfrak{b})$, luego $C \in \mathfrak{F}(\mathfrak{b})$ y por tanto $\boldsymbol{e}_{C} \in \mathfrak{b}$.

Ahora se muestra que existe una correspondencia biunívoca entre el conjunto de ideales de $R$, ideal $(R)$, y el de los filtros en I, fil(I).

Proposición 4.6 Sean $R=K^{I}$ con I un conjunto arbitrario. Entonces la aplicación

$$
\begin{array}{cl}
\mu: \operatorname{ideal}(R) & \rightarrow f i l(I) \\
\mathfrak{a} & \mapsto \mathfrak{F}(\mathfrak{a})
\end{array}
$$

Demostración. Por el Lema $4.5, \mathfrak{a}=\mathfrak{b}$ si y solo si $\mathfrak{F}(\mathfrak{a})=\mathfrak{F}(\mathfrak{b})$. Por tanto, $\mu$ está bien definida y es inyectiva. Veamos que $\mu$ es sobreyectiva. Dado un filtro $\mathfrak{F}$ de $I$, el ideal propio de $R$ asociado a $\mathfrak{F}$ es

$$
\mathfrak{a}(\mathfrak{F})=\left(\left\{\boldsymbol{e}_{C}\right\}_{C \in \mathfrak{F}}\right) R
$$

ya que

$$
\begin{aligned}
\mathfrak{a}(\mathfrak{F}(\mathfrak{a})) & =\left(\left\{\boldsymbol{e}_{C}\right\}_{C \in \mathfrak{F}(\mathfrak{a})}\right) R=\left(\left\{\boldsymbol{e}_{C}\right\}_{\boldsymbol{e}_{C} \in \mathfrak{a}}\right) R \\
& =(\{\mathfrak{a}\}) R=\mathfrak{a} .
\end{aligned}
$$

Definición 4.7 Un ultrafiltro es un filtro maximal con respecto a la relación de contenido.

Proposición 4.8 La correspondencia

$$
\mu: \operatorname{ideal}(R) \rightarrow \operatorname{fil}(I)
$$

relaciona biunívocamente los ideales maximales de $R$ con los ultrafiltros de $I$.

Demostración. Por la Proposición 3.6, todo ideal primo de $R$ es maximal. Además, por el Lema 4.5 y la Proposición 4.6, $\mu$ es una aplicación biyectiva que preserva la relación de contenido, por tanto los ideales maximales de $R$ son enviados en los ultrafiltros de $I$.

Lema 4.9 (1) Si $\left\{\mathfrak{F}_{\alpha}\right\}_{\alpha \in T}$ es una familia no vacía de filtros de $I$, entonces $\bigcap_{\alpha \in T} \mathfrak{F}_{\alpha}$ es un filtro de $I$.

(2) Si $\mathcal{C}=\left\{\mathfrak{F}_{i}\right\}_{i \in \mathbb{N}}$ es una cadena, es decir, $\left\{\mathfrak{F}_{i}\right\}_{i \in \mathbb{N}}$ es una familia no vacía de filtros de $I$ tal que $\mathfrak{F}_{i} \subset \mathfrak{F}_{i+1}$, entonces $\bigcup \mathcal{C}=\bigcup_{i \in \mathbb{N}} \mathfrak{F}_{i}$ es un filtro de $I$.

Demostración. (1) Se deduce de la Proposición 4.6 y el hecho que la intersección de ideales es un ideal.

(2) Veamos que $\bigcup_{i \in \mathbb{N}} \mathfrak{F}_{i}$ cumple las condiciones de un filtro de $I . \bigcup_{i \in \mathbb{N}} \mathfrak{F}_{i} \neq \emptyset$ ya que $\left\{\mathfrak{F}_{i}\right\}_{i \in \mathbb{N}}$ es una familia no vacía. Además si $B, C \in \bigcup_{i \in \mathbb{N}} \mathfrak{F}_{i}$, como $\mathfrak{F}_{i} \subset \mathfrak{F}_{i+1}$, existe $i$ tal que $B, C \in \mathfrak{F}_{i}$ y por tanto $B \cap C \in \mathfrak{F}_{i}$, luego $B \cap C \in \bigcup_{i \in \mathbb{N}} \mathfrak{F}_{i}$. Por último, si $C \in \bigcup_{i \in \mathbb{N}} \mathfrak{F}_{i}$ y $C \subset D$, existe $i$ tal que $C \in \mathfrak{F}_{i}$ y $C \subset D$, luego $D \in \mathfrak{F}_{i}$ y por tanto $D \in \bigcup_{i \in \mathbb{N}} \mathfrak{F}_{i}$.

Lema 4.10 Todo filtro puede extenderse a un ultrafiltro. 
Demostración. Sea $\mathfrak{F}_{0}$ un filtro en I. Supongamos $\mathbf{P}$ el conjunto de todos los filtros $\mathfrak{F}$ en $I$ tales que $\mathfrak{F} \supset \mathfrak{F}_{0}$ y consideremos el conjunto parcialmente ordenado $(\mathbf{P}, \subset)$. Si $\mathcal{C}$ es una cadena en $\mathbf{P}$, por el Lema 4.9(2), $\bigcup \mathcal{C}$ es un filtro y por tanto una cota superior de $\mathcal{C}$ en $\mathbf{P}$. Por el lema de Zorn existe un elemento maximal $\mathfrak{U}$ en $\mathbf{P}$ y por definición, $\mathfrak{U}$ es un ultrafiltro.

Proposición 4.11. [14, Theorem 7.6] Existen exactamente 22\#(I) ultrafiltros de $I$.

Ejemplo 4.12. Si $I=N$ y $K=Z /(2)$, por las Proposiciones 4.8 y 4.11 ,

$$
\#\left(\operatorname{Max}\left(K^{I}\right)\right)=2^{2^{\#(\mathbb{N})}} .
$$

Como $2^{2^{\aleph_{0}}}>\aleph_{1}$, hay una cantidad de ideales maximales de $K^{I}$ que no se pueden describir pero hay una cantidad numerable de la forma $\mathfrak{m}_{i}$.

\section{AGRADECIMIENTOS}

Los autores agradecen a la Universidad Industrial de Santander por el tiempo concedido para la investigación mediante el Proyecto de investigación titulado "Álgebras locales de dimensión finita como espacio vectorial", C-2018-03, y a los árbitros por sus comentarios y sugerencias que ayudaron a mejorar el contenido de este artículo.

\section{REFERENCIAS}

[1] P. Abellanas, Geometría Básica. Madrid: Editorial Romo S. L., 1969. 2

[2] F. Anderson and K. Fuller, Rings and $\mathrm{Ca}$ tegories of Modules, Second Edition. New York: Springer-Verlag, 1992. 2, 3, 7

[3] M. Arapovic, "Characterizations of the 0-dimensional rings", Glasnik Matematicki, vol. 18 , no. 38 , pp. $39-46,1983.7$

[4] M.F. Atiyah y I.G. Macdonald, Introducción al álgebra conmutativa. Barcelona: Editorial Reverté S. A., 1980. 4, 7
[5] N. Bourbaki, Commutative Algebra, Cap 1-7. Berlin Heidelberg: Springer-Verlag, 1989. 7

[6] P. Clark, Commutative Algebra. Giorgia: University of Giorgia, 2015. 2

[7] D. Eisenbud, Commutative Algebra, with a view toward Algebraic Geometry. New York: Springer-Verlag, 1995. 7

[8] J. Elizondo, Anillos, ideales y espectro primo. México: Universidad Nacional Autónoma de México. Disponible en: http://www. math.unam.mx/javier/caps1-2-3. pdf 4

[9] R. Gilmer and W. Heinzer, "Products of commutative rings and zero-dimensionality", Trans. Amer. Math. Soc., vol. 331, pp. 663-680, 1992. 2, 7

[10] C. Granados-Pinzón, “Álgebras finitas sobre un cuerpo. La recta proyectiva", Tesis doctoral, Dep. análisis mat., álgebra, geometría y topología, Universidad de Valladolid, Valladolid, 2015. 2

[11] C. Granados-Pinzón y W. Olaya-León, "Kálgebras finitas conmutativas con unidad", Ingeniería y Ciencia, vol. 12, no. 24, pp. 31-49, 2016. 2

[12] E. Hartmann, Planar Circle Geometries: an introduction to Moebius-, Laguerre- and Minkowski-planes, Darmstadt University of Technology, 2004. 2

[13] H. Havlicek and K. List, "A three-Dimensional Laguerre geometry and its visualization", In proceedinhs-Dresden Symposium geometry: constructive and kinematic. Institut für geometrie TU Dresden, Dresden pp. 122-129, 2003. 2

[14] T. Jech, Set theory, The third millenium editions, revised and expanded, Springer-Verlag, 2003. 9

[15] R. Levy, P. Loustaunau and J. Shapiro, "The prime spectrum of an infinite product of 
copies of Z”, Fund. Math., vol. 138, pp. 115$164,1991.2,7$

[16] O. Lezama, Cuadernos de Álgebra, No. 10: Geometría algebraica, SAC2. Departamento de Matemáticas, Universidad Nacional de Colombia, Bogotá, 2014. 4

[17] H. Matsumura, Commutative Ring Theory. Cambridge: Cambridge University Press, 1989. 7
[18] J.A. Navarro, Álgebra conmutativa básica. Extremadura: Universidad de Extremadura, 1996. 4

[19] B. Olberding and J. Shapiro, "Prime ideals in ultraproducts of commutative rings", $J$. Algebra, vol. 285, pp. 768-794, 2005. 2, 7

[20] I. Rubio y L. Acosta, "On spectral compactness of Von Neumann regular rings", Rev. Colombiana Mat., vol. 46, pp. 81-95, 2012. 5

[21] J.B. Sancho, Apuntes para una licenciatura. Salamanca: Universidad de Salamanca, 2014. 4 
